\title{
Lattice Boltzmann parallel simulation of microflow dynamics over
}

\section{structured surfaces}

\author{
Wenning Zhou ${ }^{\mathrm{a}, *}$, Yuying Yan ${ }^{\mathrm{b}}$, Xunliang Liu ${ }^{\mathrm{a}}$, Baiqian $\mathrm{Liu}^{\mathrm{a}}$ \\ ${ }^{a}$ School of Energy and Environmental Engineering, University of Science and Technology \\ Beijing, Beijing 100083, China \\ ${ }^{\mathrm{b}}$ Energy \& Sustainability Research Division, Faculty of Engineering, University of \\ Nottingham, University Park, Nottingham NG7 2RD, UK
}

*Corresponding to wenningzhou@ @ustb.edu.cn, Tel: +86 (0) 1062332730 


\begin{abstract}
In the present work, a parallel lattice Boltzmann multiphase model was developed to investigate the effects of surface structures on wettabilities and flow dynamics in a microchannel. The theory of wetting transition was firstly discussed. Then three types including triangular, rectangle and hierarchical shaped microstructures were constructed on the surface to examine the effects on wettabilities and drag reduction. It was found that flow behaviour is strongly affected by the surface morphology of the channel. The results indicated that hierarchical structures on the surface could improve the hydrophobicity significantly. For rectangular structures, they can improve the hydrophobicity with the increase of height and distance ratio $h / d$ of the structures, and the improvement will reach its optimal hydrophobicity when the value $h / d$ is over a certain value of 0.6 . Moreover, to accelerate computational speed, the Open Multi-Processing (OpenMP) was employed for the parallelization of the model. A maximum speedup of 2.95 times was obtained for 4 threads on a multi-core CPU platform.
\end{abstract}

Key words: Surface structures; Wettability; Drag reduction; Lattice Boltzmann method; Parallel computing; OpenMP

\title{
NOMENCLATURE
}

$c \quad$ lattice speed

$c_{i} \quad$ discrete particle speeds

$c_{s} \quad$ speed of sound

$f_{i} \quad$ density distribution function

$f^{e q} \quad$ equilibrium distribution function

$F_{i} \quad$ forcing term

$g$ gravitational acceleration 


$$
\begin{array}{ll}
g_{i} & \text { energy distribution function } \\
k & \text { effective thermal conductivity } \\
w_{i} & \text { weighting factor } \\
G & \text { fluid-fluid interaction strength } \\
G_{t} & \text { fluid-solid interaction strength }
\end{array}
$$

\section{Greek Symbols}

$\begin{array}{ll}\tau & \text { relaxation time } \\ \rho & \text { density } \\ \mu & \text { dynamic viscosity } \\ \nu & \text { kinetic viscosity } \\ \Omega & \text { collision operator } \\ \theta & \text { contact angle }\end{array}$

\section{Introduction}

Superhydrophobic surfaces with apparent water contact angles higher than $150^{\circ}$ and low hysteresis have received immense interest in both scientific research and industrial field over the past decade, such as self-cleaning, anti-corrosion, drag reduction, drug delivery, optical devices, microfluidic devices and so forth [1]. These surfaces with high contact angle and low contact angle hysteresis with a self-cleaning effect also exhibit low adhesion and drag reduction for fluid flow [2]. Although superhydrophobic surfaces are usually designed with low surface free energy materials, the method of chemical surface modification alone can typically lead to water contact angles of up to $120^{\circ}$. To achieve extreme values of contact angles larger than $150^{\circ}$ (near $180^{\circ}$ ), the modification on surface structure has to be utilized [3]. The effects of surface roughness on wettability have been studied for a few decades after pioneering work carried out by Wenzel [4] and Cassie-Baxter [5] who proposed theoretical models to predict the wetting behaviour of the droplet in the non-composite and composite states. The superhydrophobicity mechanism of the lotus leaf was theoretically analysed by 
Marmur [6]. It has been found that the meta-stable states in the heterogeneous wetting regime play a key role in superhydrophobicity. A review paper regarding the impacts of surface roughness on wettabilities can be found in Ref. [7]. In order to construct artificial superhydrophobic surfaces, various methods and techniques have recently been developed. With these techniques, a great number of different surface morphologies have been fabricated successfully, such as the pillar morphology [8], flowerlike structure [9], ratchet-like morphology [10], the trapezoid morphology [11], and so on. Meanwhile, numerical studies have also been carried out extensively. Gao et al. [12] proposed a model to combine both factors caused by surface structure and energy change. They claimed that the Cassie-Baxter equation should be adopted for hierarchical roughness surface. Ambrosia et al. [13] used molecular dynamics simulations to investigate the hydrophobic properties of water droplets on regular pillared surface. It should be noted their work was limited to very small length and time scale due to the expensive computational cost of molecular dynamics method. A lattice Boltzmann model was developed to study the contact angles of droplets on the surfaces with regular roughness structures [14]. Lee et al. [15] has recently developed a lattice Boltzmann model to investigate the movement of droplet on stripe-patterned surfaces. Jung et al. [16] also employed the lattice Boltzmann method to determine the optimal geometry of microstructures to achieve superhydrophobicity. Their simulation results were also compared with the results of measured wettability of fabricated micro-hierarchical metal surface. However, the previous studies mainly focused on the effect of surface structures on wettability and there are still few studies focusing on effects of the surface topography on drag reduction.

Over the past few years, the lattice Boltzmann method (LBM), a mesoscopic approach, has experienced tremendous advances and has been well accepted as a useful method to simulate various complex fluid phenomena, such as multiphase /multicomponent flows [17-19], 
electro-osmotic flow [20], micro/nano fluidics [21, 22], Magneto-hydrodynamic flows [23, $24]$, flows through porous media [25, 26], reaction-diffusion system [27, 28], and etc. Due to its kinetic nature and local dynamics, lattice Boltzmann method has several advantages over traditional numerical methods, including the physical representation of microscopic interactions, the easiness in dealing with complex geometries and parallelization of the algorithm. Recently, parallelization has become an important feature for numerical methods as high performance computing (HPC) are currently being designed for solving large-scale and complex engineering problems. The widely used parallel algorithms for LBM include multi-core CPUs [29], General Purpose GPU (GPGPU) [30] and hybrid CPU-GPU [31].

Based on our previous work on fabricating superhydrophobic surfaces and lattice Boltzmann simulating of complex fluids $[22,23,32,33]$, we extended our research to numerical investigating of structured surfaces. The objective of this study is to develop a parallel LBM model to investigate the effects of different surface topography on the wettabilities and drag reduction. The rest of this paper is organized as follows: Section 2 introduces the methodology, including the multiphase lattice Boltzmann method, the wetting transition theory and the parallel algorithm. The performance of the parallelization and simulation results on wettabilities and drag reduction are given in Section 3. Finally, the conclusions are drawn in Section 4.

\section{Methodology}

\subsection{The multiphase lattice Boltzmann method}

The pseudo-potential lattice Boltzmann model for multicomponent multiphase fluid was employed in the present study [34]. The particle distribution function (PDF) of each component of the multiphase fluid satisfies the following equation:

$$
f_{i}^{\sigma}\left(x+c_{i} \Delta t, t+\Delta t\right)-f_{i}^{\sigma}(x, t)=\Omega_{\text {coll }}^{\sigma}
$$


where $f_{i}^{\sigma}(x, t)$ is the density distribution function of component $\sigma$ and $\Omega_{\text {coll }}^{\sigma}$ is the collision operator, which has the form in the single-relaxation-time (SRT) LBM model:

$$
\Omega_{\text {coll }}^{\sigma}=-\frac{1}{\tau^{\sigma}}\left(f_{i}^{\sigma}(x, t)-f_{i}^{\sigma, e q}(x, t)\right)
$$

where $\tau^{\sigma}$ is a relaxation time which is related to its kinematic viscosity as $v^{\sigma}=c_{s}^{2}\left(\tau^{\sigma}-0.5 \Delta t\right)$. The equilibrium distribution function $f_{i}^{\sigma, e q}(x, t)$ can be calculated by the following equation:

$$
f_{i}^{\sigma, e q}=\rho_{\sigma} w_{i}\left[1+\frac{c_{i} \cdot u_{\sigma}^{e q}}{c_{s}^{2}}+\frac{\left(c_{i} \cdot u_{\sigma}^{e q}\right)^{2}}{2 c_{s}^{4}}-\frac{u_{\sigma}^{e q} \cdot u_{\sigma}^{e q}}{2 c_{s}^{2}}\right]
$$

where $w_{i}$ are weighting factors specific to the chosen lattice. For the two-dimensional ninevelocity lattice Boltzmann model (D2Q9, as seen in Fig.1) employed in this work, $w_{i}$ are 4/9, $1 / 9$ and $1 / 36$, for $i=0,1-4,5-8$, respectively [35]. $\rho_{\sigma}$ is the density of component $\sigma . c_{s}$ is the sound speed. $c_{i}$ is the discrete velocities which are defined as:

$$
c_{i}=\left\{\begin{array}{l}
(0,0), i=0 \\
c\left(\cos \theta_{i}, \sin \theta_{i}\right),\left(\theta_{i}=(i-1) \pi / 2, i=1,2,3,4\right) \\
\sqrt{2} c\left(\cos \theta_{i}, \sin \theta_{i}\right),\left(\theta_{i}=(i-5) \pi / 2+\pi / 4, i=5,6,7,8\right)
\end{array}\right.
$$

where $c_{i}$ is the particle streaming speed and determined by $c=\Delta x / \Delta t . \Delta x, \Delta t$ are the lattice spacing and time step, respectively. The relation between $c_{s}$ and $c$ can be expressed as $c_{s}=c / \sqrt{3}$. The macroscopic density and momentum of the $\sigma$ th component are defined as:

$$
\begin{gathered}
\rho_{\sigma}=\sum_{i} f_{i}^{\sigma} \\
\rho_{\sigma} u_{\sigma}=\sum_{i} f_{i}^{\sigma} c_{i}
\end{gathered}
$$

The equilibrium velocity $u_{\sigma}^{e q}$ in Eq. (3) is defined as:

$$
\rho_{\sigma} u_{\sigma}^{e q}=\rho_{\sigma} u^{\prime}+\tau_{\sigma} F_{\sigma}
$$


where $u^{\prime}$ is an effective velocity and $F_{\sigma}=F_{c, \sigma}+F_{a d s, \sigma}+F_{e, \sigma}$ is the total force acting on the $\sigma$ th component including fluid-fluid interaction $F_{c, \sigma}$, fluid-solid interaction $F_{a d s, \sigma}$ and external force $F_{e, \sigma}$. To conserve momentum in the absence of forces, $u^{\prime}$ should satisfy:

$$
u^{\prime}=\sum_{\sigma} \frac{\rho_{\sigma} u_{\sigma}}{\tau_{\sigma}} / \sum_{\sigma} \frac{\rho_{\sigma}}{\tau_{\sigma}}
$$

In pseudo-potential model, the fluid-fluid interaction can be expressed as:

$$
F_{c, \sigma}(x)=-\varphi(x) \sum_{x^{\prime}} \sum_{\bar{\sigma}} G_{\sigma \bar{\sigma}}\left(x, x^{\prime}\right) \varphi\left(x^{\prime}\right)\left(x^{\prime}-x\right)
$$

where $G_{\sigma \bar{\sigma}}\left(x, x^{\prime}\right)$ is a parameter that controls the strength of the interaction force. $\varphi(x)$ is the "effective density" of the $\sigma$ th component defined as a function of the local particle density. Different forms of $\varphi(x)$ lead to different equations of state. $G_{\sigma \bar{\sigma}}=G$ for $\left|x^{\prime}-x\right|=c ; G_{\sigma \bar{\sigma}}=G / 4$ for $\left|x^{\prime}-x\right|=\sqrt{2} c$ and $G_{\sigma \bar{\sigma}}=0$ for otherwise. The most distinctive feature of the pseudopotential lattice Boltzmann method is that the phase segregation between different phases can emerge automatically as a result of the particle interactions.

Martys and Chen [36] proposed to introduce the interaction force to describe the interaction between a fluid and a wall. The interaction force is expressed as:

$$
\begin{aligned}
& F_{a d s, \sigma}(x)=-G_{a d s} \varphi(x) \sum_{i} s\left(x^{\prime}\right)\left(x^{\prime}-x\right) \\
& G_{a d s}=\left\{\begin{array}{l}
G_{t},\left|x^{\prime}-x\right|=c \\
G_{t} / 4,\left|x^{\prime}-x\right|=\sqrt{2} c \\
0, \text { otherwise }
\end{array}\right.
\end{aligned}
$$

where the parameter $G_{t}$ determines the fluid-solid interaction strength. $s\left(x^{\prime}\right)=0$ or 1 indicates fluid and solid node, respectively. The surface wetting characteristics can be controlled by adjusting the fluid-solid interaction strength $G_{t}$. In this study, the fluid-solid interaction strength $\left|G_{t}\right|$ within the range 0.01-0.3 referring hydrophobic surfaces is investigated. 


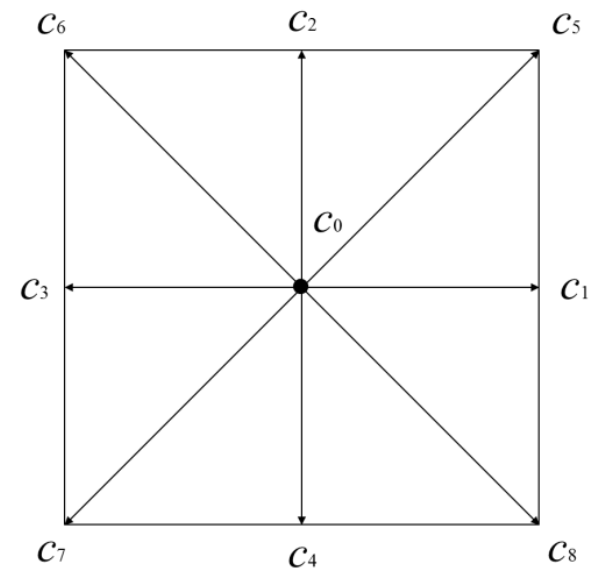

Fig. 1. Typical two dimensional lattice Boltzmann model (D2Q9)

\subsection{Wetting transition}

In Young's wetting state, when a liquid-gas interface meets a flat partial wetting surface, the contact angle mearsured in the liquid, can be calculated from a balance of surface tension forces at the contact line [37]:

$$
\cos \theta_{Y}=\left(\sigma_{S G}-\sigma_{S L}\right) / \sigma_{L G}
$$

where $\sigma_{S G}$ and $\sigma_{S L}$ are the solid-gas and solid-liquid surface tension, respectively.

For roughness surfaces, modified versions of Young's equation are need to interpret the pratical contact angles. Wenzel proposed a model where tha apparent contact angle depends on a roughness parameter $r$ and the contact angle on a flat surface [4]:

$$
\cos \theta_{W}=r \cos \theta_{Y}
$$

where the roughness parameter $r$, also referred as roughness area ratio, is denoted as the ratio of the actual surface-area over the projected area of the structures. As Wenzel assumed the water would penetrate into the grooves on the rough surface when it spead on the surface, the Wenzel's equation relates to the homogeneous wetting regime [38]. If air is entrapped inside the grooves of roughness structures, it turns to Cassie-Baxter wetting state, 
in which the liquid only contacts the solid through the top of the roughness, on a fraction $f$ [39]:

$$
\cos \theta_{C B}=f\left(\cos \theta_{Y}+1\right)-1
$$

where $f$ is the fraction of the solid-liquid interface, $(1-f)$ is the fraction on the gas-liquid interface. The Cassie-Baxter state is related to the heterogeneous wetting regime.

According to the thermodynamically stability, the droplet prefers the state with a lower free energy [38]. There is a threhold Young's angle $\theta_{C}$ [40]. If $\theta_{Y}>\theta_{C}$, the droplet can keep a stable Cassie-Baxter state; On the other hand, if $\theta_{Y}<\theta_{C}$, the droplet prefers to stay in Wenzel state. In the present work, the wetting behaviours have been properly simulated by setting fluid-solid interaction strength parameter and using proper boundary conditions, which will be discussed in detail in the next section. Previous research also indicated that wetting transition between Wenzel and Different Cassie-Baxter state can be predited by using lattice Boltzmann method [14]. Contact angles with different states are illustrated in Fig. 2.

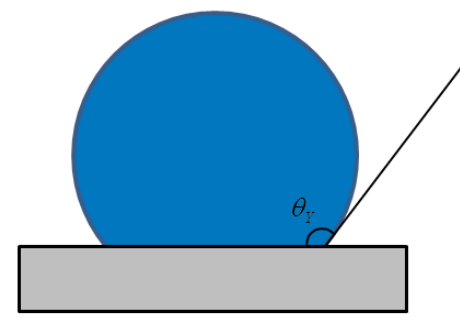

(a)

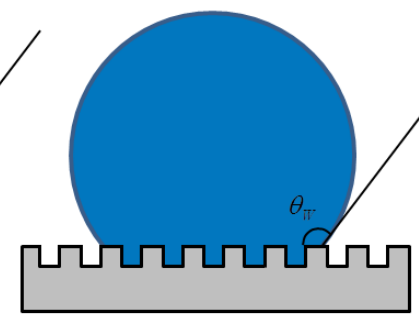

(b)

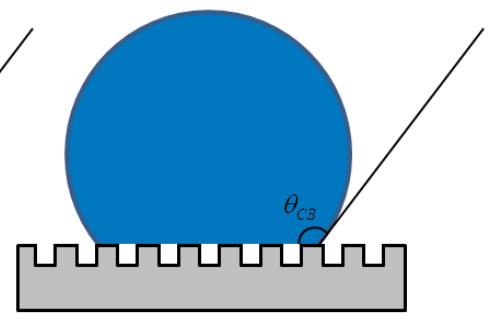

(c)

Fig. 2. Contact angles of the droplet on the surfaces: (a) Young's angle on the flat surface (b) in Wenzel state (c) in Cassie-Baxter state

\subsection{Multi-core CPU programming for LBM model}


The most time demanding in the LBM model are the collision and streaming steps. Only one CPU with single thread can be used in a serial code. As the collision step is purely local and the streaming step only requires the data of the neighbouring nodes, the LBM model is very suitable for parallel computing. To use multi-threads in the simulation, OpenMP (Open Multi-Processing) was employed to achieve the parallelization of the proposed model. The parallel implementation is demonstrated in Fig. 3. It should be noted that the variables in the parallel region should be carefully defined to avoid race condition. Part of the source code for parallelized collision step of the proposed LBM model is shown in Fig. 4.

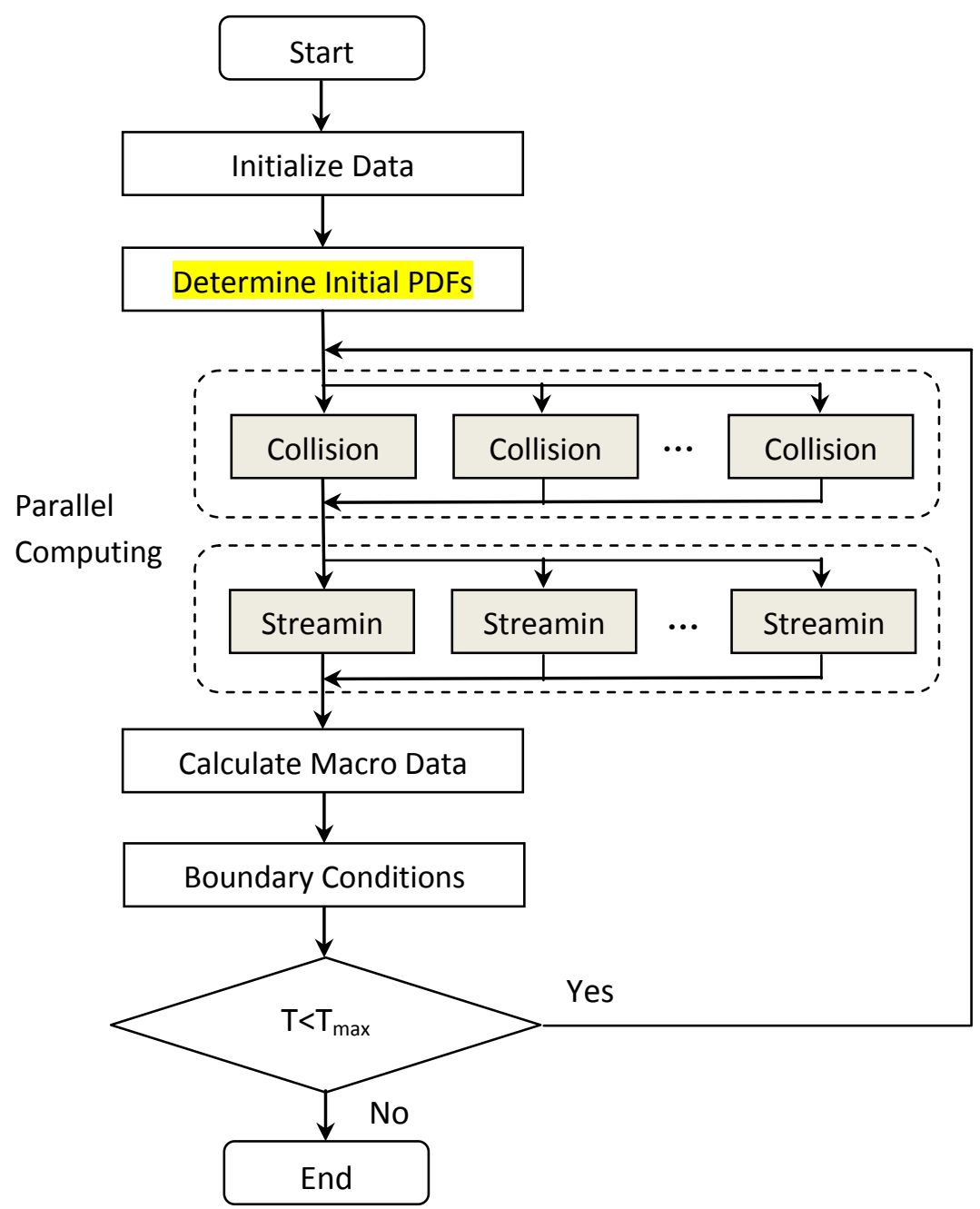

Fig. 3. Flowchart of the parallel LBM model 


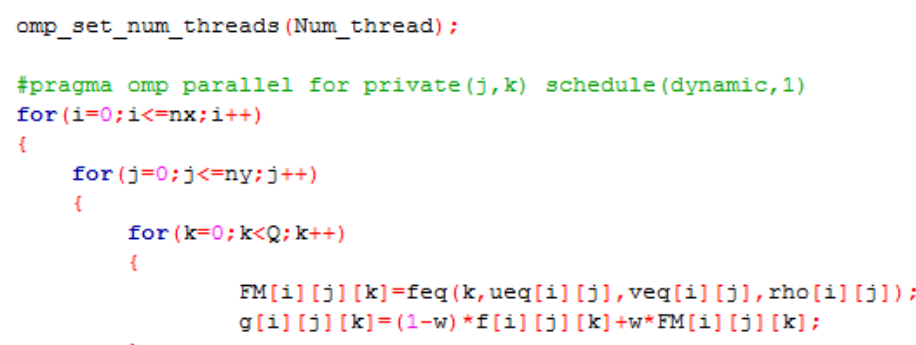

Fig. 4. The source code for the parallelized collision step

\section{Results and discussion}

\subsection{Performance of the proposed parallel computing model}

To evaluate the effectiveness of the proposed parallel model, a series of simulation tests were carried out on a DELL PC with Intel multi-core CPU i7-4790, 3.60GHz. 1-4 threads were utilized in the simulations. Table 1 shows the results of computing times with different numbers of thread used in the simulation. It is found that a maximum speedup of 2.42 times was achieved by using 4 threads. Fig. 5 displays that the speedup increased with an increasing mesh size. The $\mathrm{X}$-axis represents the mesh size of the height of the channel (in lattice unit). The length of the channel is fixed at 1500. It can be seen from this figure that the acceleration further increased as the computational domain increased. A speedup of 2.95 times was obtained when using 4 CPUs at the $1500 \times 120$ computational domain. It is indicated that the efficiency for a larger or three dimensional computational domain of a more complex physical phenomenon could be significantly improved when more CPU cores on high performance computing systems are utilized. 
Table 1 Computing times for 2000 time intervals on a DELL PC with multi-core CPU, $1500 \times 50$ grids

\begin{tabular}{ccc}
\hline Threads & Time/s & Speedup \\
\hline 1 & 103.091 & 1 \\
2 & 60.666 & 1.699 \\
3 & 49.411 & 2.086 \\
4 & 42.603 & 2.420 \\
\hline
\end{tabular}

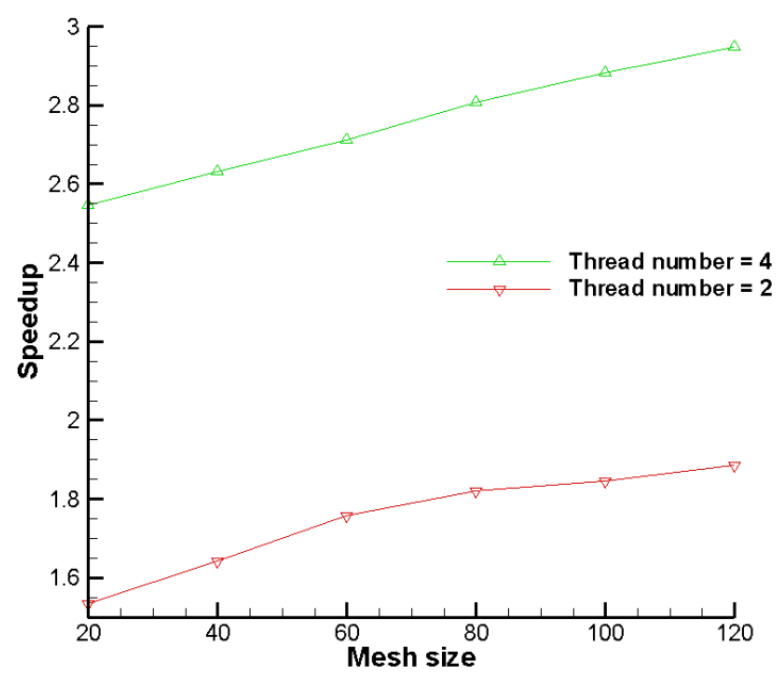

Fig. 5. The acceleration as a function of mesh size (lattice unit, height of the channel)

\subsection{Evaluation of the proposed lattice Boltzmann model}

In order to validate the proposed multiphase LBM model, we first simulated a droplet in an unbounded domain. In the simulation, a series of droplets with different radii were initially placed in the middle of a computational domain which was discretized into $100 \times 100$. Periodic boundary conditions were employed on four sides of the computational domain. After the equilibrium state was achieved, the pressure difference and radii of the droplets can be obtained. Fig. 6 demonstrates that the results of numerical simulation agree quite well with Laplace's law which could be written as:

$$
\Delta p=\frac{\sigma}{R}
$$


The values of pressure and radius are very sensitive to the final results because they are relatively small. Therefore, carefulness is needed when choosing these values in simulating cases. The values of the pressure should be a constant theoretically. However, since the thickness of the interface is finite and both phases exist near the interface, the values of pressure vary near the interface. Therefore, the values of pressure are taken away from the interface where the pressure is almost a constant. As can be seen from Fig. 6, the slopes of the lines are different with different fluid-fluid interaction strength. In other words, different surface tensions could be achieved by varying the parameter of interaction strength parameter in the proposed model.

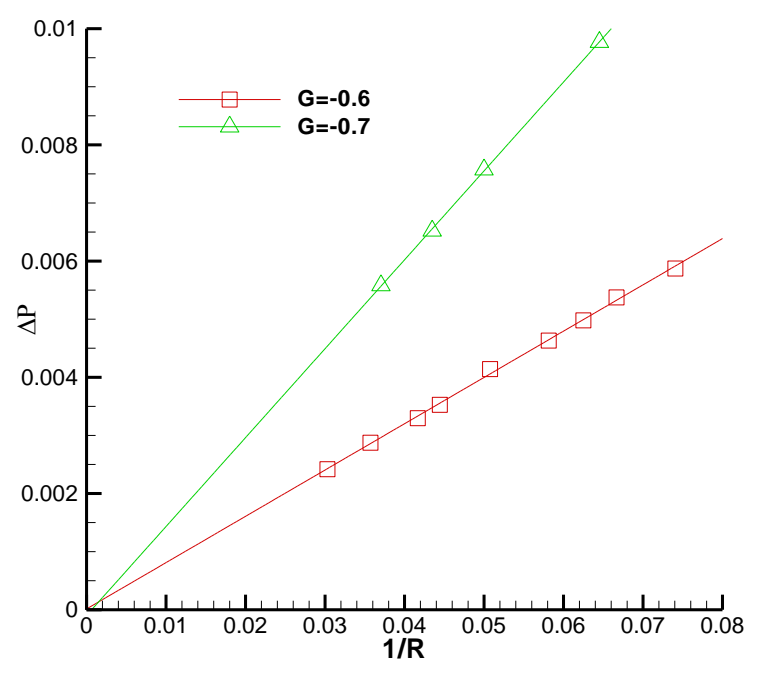

Fig. 6. The pressure difference as a function of its curvature with different fluid-fluid interaction strength

\subsection{Evaluation on surface wettabilities}

In this section, the wettabilities of a single droplet on different surfaces were investigated. The droplet was initially placed on the surfaces. After equilibrium status was achieved, different contact angles were presented on different surfaces. Fig. 7 demonstrates different contact angles between flat and rough surfaces with rectangular morphology which have the 
same surface energy. Fig. 7a has a contact angle of $119.8^{\circ}$ on flat surface, while $129.6^{\circ}$ in rough surface with rectangle morphology, as shown in Fig. 7b. It is observed that surface morphology could have a positive effect on improving contact angles of the surface.

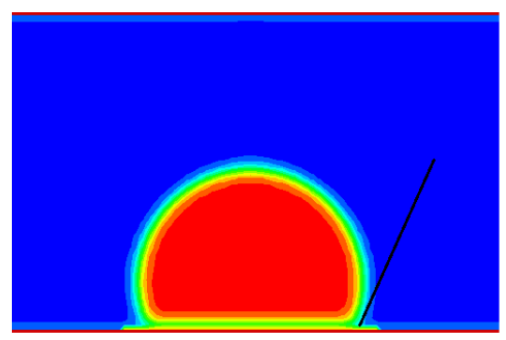

(a)

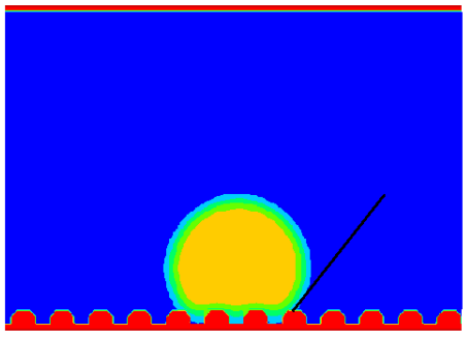

(b)

Fig. 7. Contact angles on flat surface (a) and rough surface with rectangle morphology (b)

Fig. 8 presents the result of the contact angles as a function of the fluid-solid interaction strength $G_{t}$. It can be seen that when $\left|G_{t}\right|<0.3$, the contact angle is larger than $90^{\circ}$, which is consistent with the analytical result in [41]. It means that the hydrophobic surfaces could be obtained by applying $\left|G_{t}\right|<0.3$. Fig. 9 displays the comparison of the three kinds of surfaces, i.e., flat surface, rough surfaces with rectangular and hierarchical morphology, respectively. The results show that for certain hydrophobic surfaces with the fluid-solid interaction strength $0.15<\left|G_{t}\right|<0.3$, surface roughness have an evident effect on increasing contact angles, thus enhancing the surface hydrophobicity. Overall the surface with hierarchical morphology has the most profound effects. For instance, the contact angle on flat surface is $148.0^{\circ}$ $\left(\left|G_{t}\right|=0.15\right)$, while it was increased to $173.3^{\circ}$ on the surface with hierarchical morphology. It is also observed that the effects of hierarchical morphology on increasing contact angles weaken for those surfaces are less hydrophobic, i.e., the fluid-solid interaction strength $\left|G_{t}\right|>0.25$. It should be noted that the sizes of the morphology may have different effects on changing the wettabilities which will be discussed in the next section. 


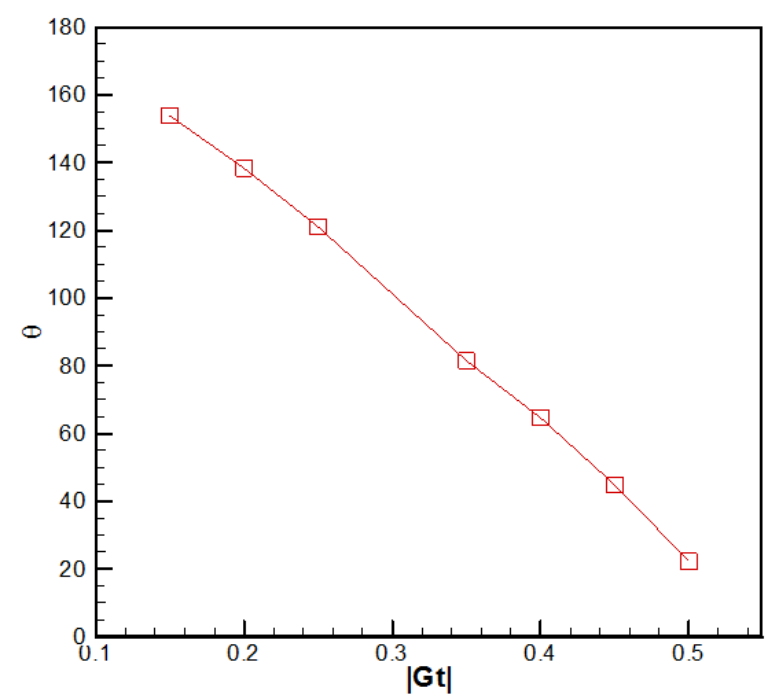

Fig. 8. The contact angle as a function of $\left|G_{t}\right|, G=-0.6$

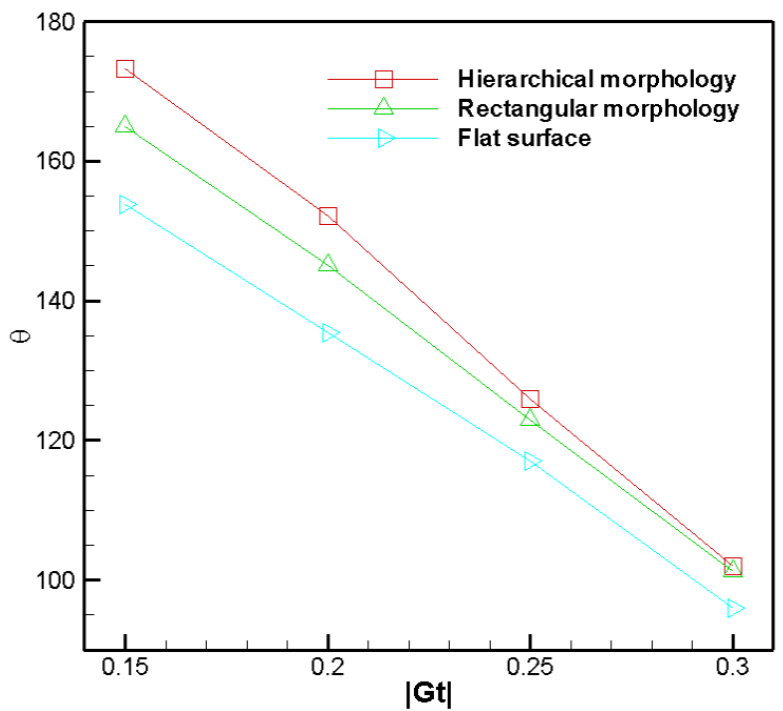

Fig. 9. Contact angels on different surfaces with different morphology

\subsection{Simulation for fluid dynamics in microchannels}

\subsubsection{Fluid flow in smooth channel}

In the present study, a uniform of $1500 \times 50$ in lattice unit was applied representing $1500 u m \times 50 u m$ microchannel. To focus on flow dynamics in the channel and avoid the entrance and exit effects, we divided the channel into two parts along the channel direction. 
The first part of channel was filled with fluid with a fixed velocity $u$, and we focused the flow dynamics at the rest of the channel. The fluid was set with a fixed velocity $u$. For boundary conditions in the proposed LBM model, periodic boundary conditions were employed at the inlet and outlet, while half-way bounce back boundary conditions were utilised at the top and bottom of the wall [42]. Fluid-fluid interaction strength parameter in this study was set at a fixed value of -0.6 throughout the simulation cases, i.e., fixed surface tension. As for fluidsolid interaction strength parameter $\left|G_{t}\right|$, a range of 0.01-0.3 was investigated. Fig. 10 displays the flows in the channel at $t=2000$ in lattice time with different fluid-solid interactions. It is observed that a gas layer is formed along the channel for $\left|G_{t}\right|=0.01$ which helps reduce the resistance from the channel. Therefore, it shows smallest resistance and longest flow length in a certain time period. As the fluid-solid interaction strength parameter $\left|G_{t}\right|$ increases, the microchannel becomes less hydrophobic. It should be noted that we assume the microchannel is more hydrophobic when it has a longer flow length in our simulation cases.

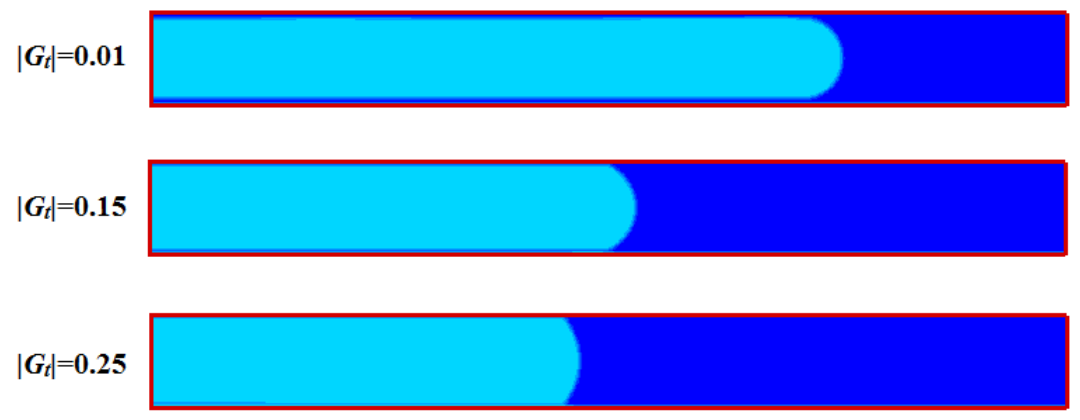

Fig. 10. Flow length in the microchannel with different interaction strength, $t=2000$

\subsubsection{Fluid flow in the channel with surface structures}

As discussed before, surface structures play an important role on influencing fluid flow dynamics. The effects of the types and sizes of surface structures were investigated in this section. Triangular, rectangle and hierarchical surface structures were created along the 
microchannel, as shown in Fig. 11. For all simulation cases, the sizes of the surface structures are set at $a=4, d=6$ (in lattice unit, similarly hereinafter). The height for triangular structures is $h=2$, while they are set at $h=5$ for rectangle and hierarchical ones. For hierarchical structures, the sizes for the second layer structure are $a^{\prime}=1, b=2, h^{\prime}=3$. The effect of rectangle surface structures on flow length at $t=3000$ is displayed in Fig. 12. It is found that the roughness helps reduce the resistance of the channel, hence increase the flow length. The simulation results also indicate that the increase of flow length with $\left|G_{t}\right|=0.1$ is much less than that of $\left|G_{t}\right|=0.2$. It means that roughness does not have an obvious impact on the channels those already possess superhydrophobic characteristics. Surface structures can improve its hydrophobicity, while they do not have an obvious impact on superhydrophobic channels, which is consistent with previous experimental work [43]. It can be seen from the figure that rectangle surface structures have the effects of helping improve the surface hydrophobicity, especially for the hydrophobic surface with fluid-solid interaction strength $0.15<\left|G_{t}\right|<0.25$.

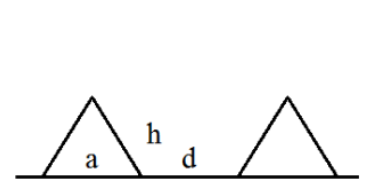

(a)

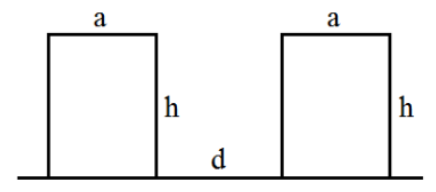

(b)

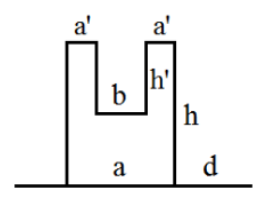

(c)

Fig. 11. The (a) triangular, (b) rectangle and (c) hierarchical structures on the channel 


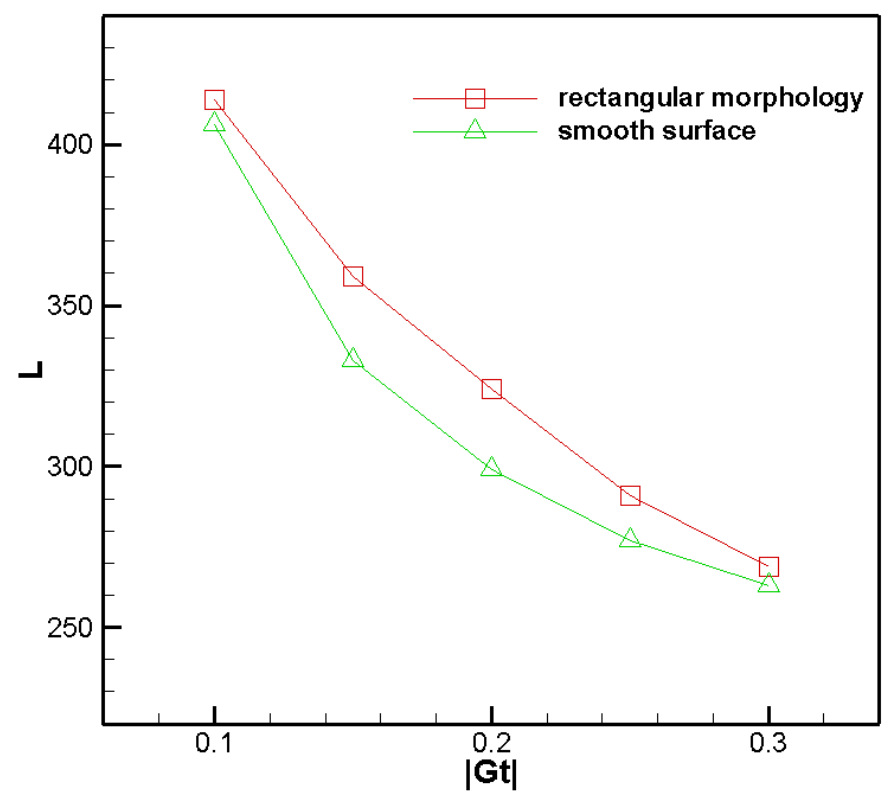

Fig. 12. The effect of rectangle surface structures on the flow length

To further investigate the effects of surface structures' size on hydrophobicity, the ratio of the rectangle roughness height and distance $h / d$ is introduced. Fig. 13 illustrates the effect of rectangle structures with different $h / d$ on the hydrophobic channel $\left(\left|G_{t}\right|=0.15\right)$. It is observed that the surface structures contribute to the hydrophobicity for most $h / d$ values. However, when the ratio is smaller than 0.3 , the surface structures play a role of resistance, as shown region $\mathrm{A}$ in the figure. As the increase of the ratio $h / d$, the flow length increases rapidly. However when the ratio $h / d>0.6$, the flow length almost remains a constant as shown region $\mathrm{C}$ in the figure. For the ratios falls in region $\mathrm{B}$, the rectangle structures on the surface help improve the surface hydrophobicity of the channel. The hydrophobicity reaches its maximum when $h / d$ is around 0.6 for rectangle surface structures. An increasing ratio of $h / d$ thereafter will not help improve the hydrophobicity further. The results could be helpful for designing superhydrophobic surfaces by patterning surface microstructures. 


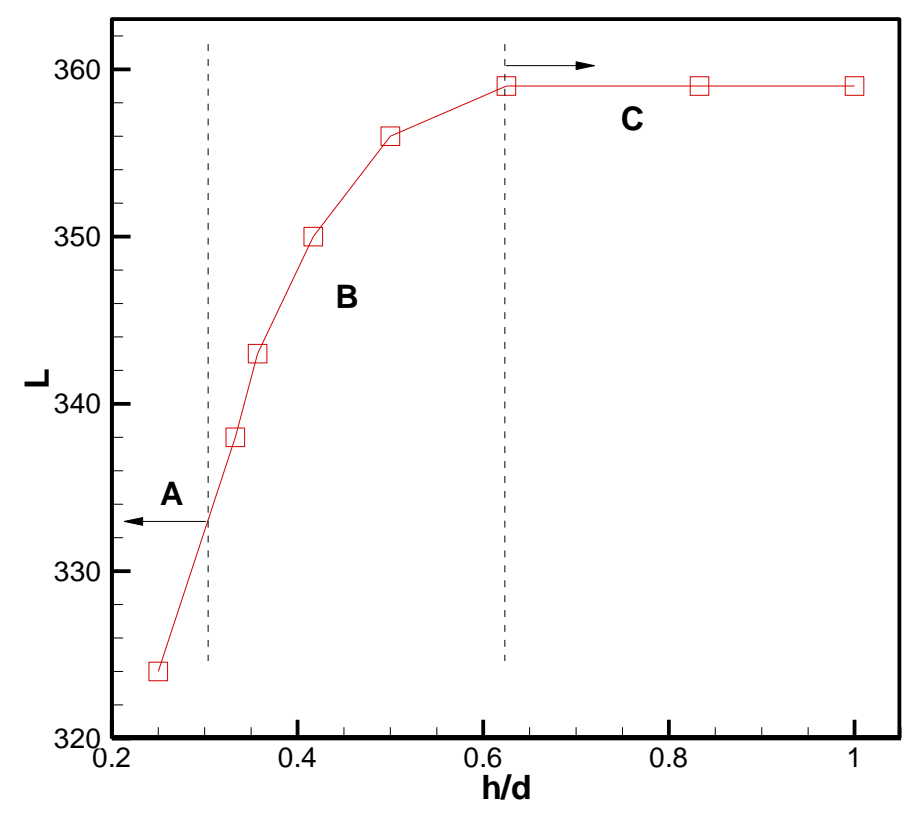

Fig. 13. Flow length in the channel with rectangle surface structures as a function of the ratio $h / d,\left|G_{t}\right|=0.15$

To achieve further superhydrophobicity, hierarchical roughness is usually designed on the surface of channel, as shown in Fig 11. Fig. 14 displays the flow lengths in different microchannels with different morphologies. As can be seen from the figure, the flow length in the channel with hierarchical morphology is longer than that with rectangular morphology. It indicates that the microchannel with hierarchical morphology is more hydrophobic than that with rectangular morphology $(h / d=5 / 6)$. Comparisons of the effects of rough channel with triangle, rectangle and hierarchical surface structures on hydrophobicity are shown in Fig. 15. It is found that the hierarchical surface structures have the most significant effect on the hydrophobicity when the fluid-solid interaction strength parameter falls into $0.1<\left|G_{t}\right|<0.2$. Hierarchical surface structures have a more evident improvement on hydrophobicity compared with other rectangular surface structures in this region. Compared with the smooth channel, the triangular surface morphology does not have an obvious effect on changing hydrophobicity. For those channels already with high hydrophobicity $\left(\left|G_{t}\right|=0.1\right)$, triangular 
surface structures may have an effect of reducing the hydrophobicity. It should be noted that when fluid-solid interaction strength $\left|G_{t}\right|>0.2$, the effects of hierarchical structures on improving hydrophobicity decrease dramatically, and eventually they will play a role of resistance of the channel. With these rules in mind, superhydrophobic channels could be created by patterning proper hierarchical structures on hydrophobic channels.

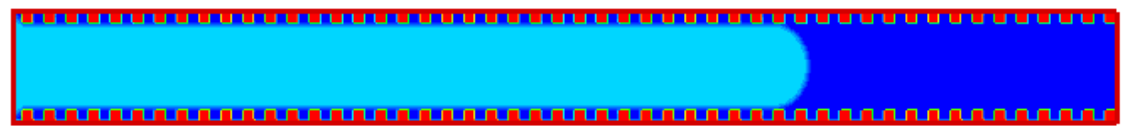

(a)

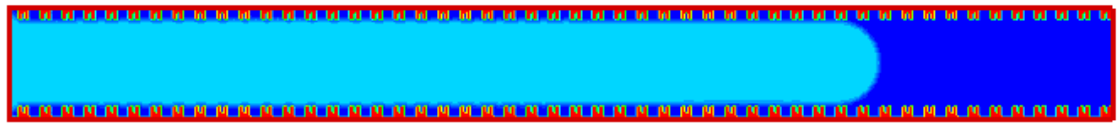

(b)

Fig. 14. Flow length in microchannels with (a) rectangular morphology, (b) hierarchical morphology, $\left|G_{t}\right|=0.15, t=4000$

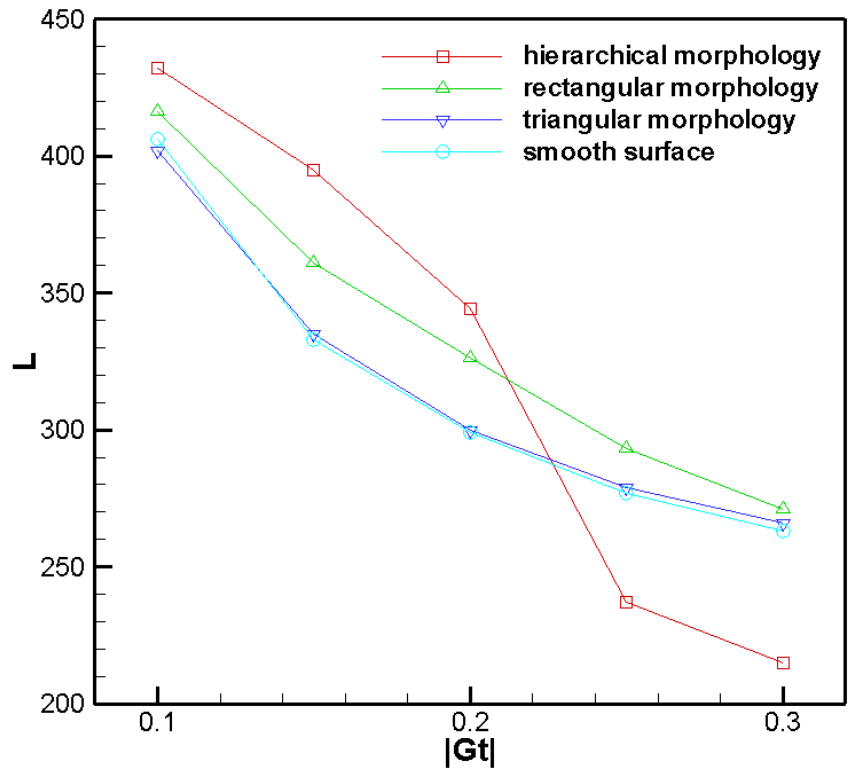

Fig. 15. Flow length in channels with different morphology 
To investigate drag reduction effect, the frictional resistance coefficient is introduced by $c_{f}=2 \Delta p d\left(|u|^{2} \rho l\right)$. The local frictional resistance coefficient at $t=2000$ and $x=L / 2$ in four kinds of channels is displayed in Fig. 16. The horizon abscissa 1 stands for the channel with the hierarchical surface structures; 2,3 represent $h / d=5 / 6, h / d=4 / 6$ rectangular surface structures, respectively; 4 denotes the channel with triangular surface structures. It can be seen from the figure that the channel with the hierarchical surface structures has the smallest frictional resistance coefficient. It indicates that the pressure drop in the channel with hierarchical roughness is the smallest compared with other rough channels.

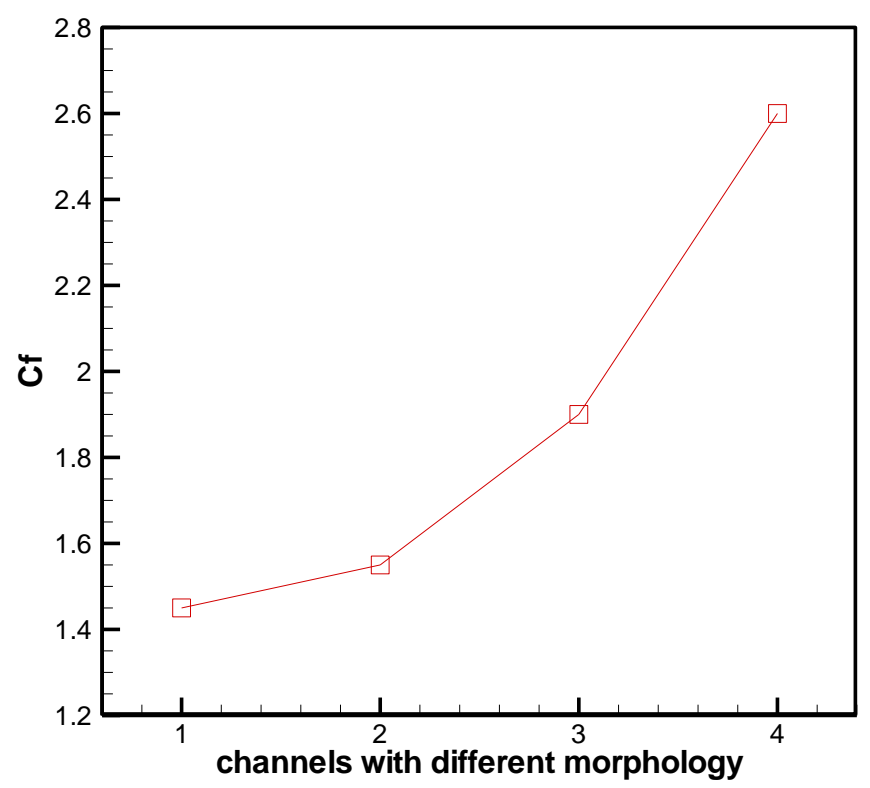

Fig. 16. The frictional resistance coefficient in different microchannels with different morphology

\section{Conclusions}

In the present work, a parallel lattice Boltzmann method was developed to investigate the effects of surface structures on the wettabilities and drag reduction of the microchannel. The theory of wetting transition and mechanism of drag reduction were analysed. We have 
discussed how the Wenzel and Cassie-Baxter equations determine the homogeneous and heterogeneous wetting. Multi-core CPU programming was introduced for the parallelization of the LBM model. A maximum speedup of 2.95 times was achieved for 4 threads on a multicore CPU i7-4790 platform. In addition, triangular, rectangular and hierarchical surface microstructures were then constructed on the surface of the microchannel. For rectangular structures, it is found that the ratio of height and the distance $h / d$ has a great effect on hydrophobicity of the surfaces. As the ratio increases, the surface morphology could help increase the hydrophobicity. However, when the ratio increases further, i.e., $h / d>0.6$, the surface morphology does not have an evident effect on improving hydrophobicity. For hierarchical surface structures, the simulation results show that they have the most pronounced effects on improving hydrophobicity and drag reduction of the channel compared with triangular and rectangular surface structures. The results could provide helpful information for the design and optimization of superhydrophobic surfaces by patterning surface microstructures.

\section{Acknowledgements}

This work is supported by the Fundamental Research Funds for the Central Universities (Grant no. FRF-TP-15-081A1) and National Science Foundation of China (Grant no. $51676013)$.

\section{References}

[1] Marmur A. The lotus effect: superhydrophobicity and metastability. Langmuir. 2004;20:3517-9.

[2] Bhushan B, Jung YC. Natural and biomimetic artificial surfaces for superhydrophobicity, selfcleaning, low adhesion, and drag reduction. Progress in Materials Science. 2011;56:1-108.

[3] Blossey R. Self-cleaning surfaces - virtual realities. Nature Materials. 2003;2:301-6.

[4] Wenzel RN. RESISTANCE OF SOLID SURFACES TO WETTING BY WATER. Industrial \& Engineering Chemistry. 1936;28:988-94. 
[5] Cassie ABD, Baxter S. Wettability of porous surfaces. Transactions of The Faraday Society. $1944 ; 40$.

[6] Marmur A. The Lotus Effect: Superhydrophobicity and Metastability. Langmuir. 2004;20:3517-9.

[7] Quéré D. Wetting and Roughness. Annual Review of Materials Research. 2008;38:71-99.

[8] Zhang H, Li W, Liu H, Cui D. Thermodynamic analysis on superhydrophobicity based on the design of a pillar model. Soft Matter. 2012;8:10360-9.

[9] Guo F, Su X, Hou G, Li P. Bioinspired fabrication of stable and robust superhydrophobic steel surface with hierarchical flowerlike structure. Colloids and Surfaces A: Physicochemical and Engineering Aspects. 2012;401:61-7.

[10] Sheng X, Zhang J. Directional motion of water drop on ratchet-like superhydrophobic surfaces. Applied Surface Science. 2011;257:6811-6.

[11] Moradi S, Englezos P, Hatzikiriakos SG. Contact Angle Hysteresis of Non-Flattened-Top Micro/Nanostructures. Langmuir. 2014;30:3274-84.

[12] Gao N, Yan Y. Modeling Superhydrophobic Contact Angles and Wetting Transition. Journal of Bionic Engineering. 2009;6:335-40.

[13] Ambrosia MS, Ha MY, Balachandar S. The effect of pillar surface fraction and pillar height on contact angles using molecular dynamics. Applied Surface Science. 2013;282:211-6.

[14] Yagub A, Farhat H, Kondaraju S, Singh T. A lattice Boltzmann model for substrates with regularly structured surface roughness. J Comput Phys. 2015;301:402-14.

[15] Lee C, Lyu S, Park JW, Hwang W. Lattice Boltzmann simulation of the movement of droplets on stripe-patterned surfaces having different wettability. Adv Eng Softw. 2016;91:44-50.

[16] Jung $M$, Kim T, Kim H, Shin R, Lee J, Lee J, et al. Design and fabrication of a large-area superhydrophobic metal surface with anti-icing properties engineered using a top-down approach. Applied Surface Science. 2015;351:920-6.

[17] Yan YY, Zu YQ. A lattice Boltzmann method for incompressible two-phase flows on partial wetting surface with large density ratio. J Comput Phys. 2007;227:763-75.

[18] Stensholt S, Øien A. Lattice Boltzmann simulations of the motion induced by variable surface tension. Adv Eng Softw. 2011;42:944-53.

[19] Zhang $M, X u K$, He $Y$, Jivkov AP. Pore-scale modelling of 3D moisture distribution and critical saturation in cementitious materials. Construction and Building Materials. 2014;64:222-30.

[20] Wang M, Wang JK, Li ZX. Lattice Poisson-Boltzmann simulations of electro-osmotic flows in microchannels (vol 296, pg 729, 2006). J Colloid Interf Sci. 2006;300:446-.

[21] Suga K, Takenaka S, Ito T, Kaneda M, Kinjo T, Hyodo S. Evaluation of a lattice Boltzmann method in a complex nanoflow. Phys Rev E. 2010;82:-.

[22] Zhou WN, Yan YY, Xu JL. A lattice Boltzmann simulation of enhanced heat transfer of nanofluids. Int Commun Heat Mass. 2014;55:113-20.

[23] Zhou WN, Yan YY. Numerical Investigation of the Effects of a Magnetic Field on Nanofluid Flow and Heat Transfer by the Lattice Boltzmann Method. Numerical Heat Transfer, Part A: Applications. 2015;68:1-16.

[24] Dellar PJ. Lattice Boltzmann magnetohydrodynamics with current-dependent resistivity. J Comput Phys. 2013;237:115-31.

[25] Zhang M, Ye G, Breugel Kv. Microstructure-based modeling of permeability of cementitious materials using multiple-relaxation-time lattice Boltzmann method. Comp Mater Sci. 2013;68:142-51. [26] Grucelski A, Pozorski J. Lattice Boltzmann simulations of heat transfer in flow past a cylinder and in simple porous media. Int J Heat Mass Tran. 2015;86:139-48.

[27] Zhang J, Yan G. Lattice Boltzmann model for the bimolecular autocatalytic reaction-diffusion equation. Appl Math Model. 2014;38:5796-810.

[28] Zhang M, Ye G, van Breugel K. Modeling of ionic diffusivity in non-saturated cement-based materials using lattice Boltzmann method. Cement and Concrete Research. 2012;42:1524-33. 
[29] Puurtinen TA, Toivanen JI, Mattila K, Hyväluoma J, Nash RW, Coveney PV, et al. Coupling of lattice-Boltzmann solvers with suspended particles using the MPI intercommunication framework. Adv Eng Softw.

[30] Habich J, Zeiser T, Hager G, Wellein G. Performance analysis and optimization strategies for a D3Q19 lattice Boltzmann kernel on nVIDIA GPUs using CUDA. Adv Eng Softw. 2011;42:266-72.

[31] Ye Y, Li K, Wang Y, Deng T. Parallel computation of Entropic Lattice Boltzmann method on hybrid CPU-GPU accelerated system. Comput Fluids. 2015;110:114-21.

[32] Gao N, Yan YY, Chen XY, Mee DJ. Superhydrophobic surfaces with hierarchical structure. Materials Letters. 2011;65:2902-5.

[33] Gao N, Yan Y, Chen X, Mee DJ. Nanoparticle Induced Morphology and Hydrophilicity of Structured Surfaces. Langmuir. 2012.

[34] Shan XW, Chen HD. Lattice Boltzmann Model for Simulating Flows with Multiple Phases and Components. Phys Rev E. 1993;47:1815-9.

[35] Aidun CK, Clausen JR. Lattice-Boltzmann Method for Complex Flows. Annu Rev Fluid Mech. 2010;42:439-72.

[36] Martys N, Chen H. Simulation of multicomponent fluids in complex three-dimensional geometries by the lattice Boltzmann method. Phys Rev E. 1996;53:743-50.

[37] Young T. An essay on the cohesion of fluids. Philosophical Transactions of the Royal Society of London. 1805:65-87.

[38] Marmur A. Wetting on hydrophobic rough surfaces: to be heterogeneous or not to be? Langmuir. 2003;19:8343-8.

[39] Cassie A, Baxter S. Wettability of porous surfaces. Transactions of the Faraday Society. 1944;40:546-51.

[40] Zheng Q-S, Yu Y, Zhao Z-H. Effects of hydraulic pressure on the stability and transition of wetting modes of superhydrophobic surfaces. Langmuir. 2005;21:12207-12.

[41] Sukop MC, Thorne DT. Lattice Boltzmann modeling: An introduction for geoscientists and engineers: Springer Verlag; 2006.

[42] Ziegler DP. Boundary-Conditions for Lattice Boltzmann Simulations. J Stat Phys. 1993;71:1171-7.

[43] Gao N, Yan Y, Chen X, Zheng X. Superhydrophobic Composite Films Based on THS and Nanoparticles. Journal of Bionic Engineering. 2010;7:S59-S66. 